\title{
Values and validity of fetal parameters by ultrasound and Doppler as markers of fetal lung maturity
}

Radwa A. Kandil ${ }^{1}$, Al shymaa Z. El shahawy², Mohamed H. El Shafiey² and Reda A. Alarabawy ${ }^{2 *}$

\begin{abstract}
Background: In this study, we combined ultrasound and Doppler parameters to conclude the most accurate and applicable method for fetal lung maturity assessment. The purpose is to reduce risk of neonatal respiratory distress by assessment of fetal lung maturity through ultrasound and Doppler instead of amniocentesis.
\end{abstract}

Results: A total of 120 women were included. The ultrasound examination was performed at a gestational age ranging between 30 and 37 weeks and delivery occurred within $72 \mathrm{~h}$ from ultrasound. After birth, 19 fetuses were diagnosed with RDS.

The ultrasound parameters assessed for fetal lung maturity showed varying sensitivity and specificity but presence of proximal tibial epiphyses showed the highest sensitivity (91\%) and specificity (95\%) followed by the presence of distal femoral epiphyses with $90 \%$ sensitivity and $84 \%$ specificity. The placental grade III maturity showed a sensitivity of $81 \%$ and $74 \%$ specificity followed by the presence of the amniotic fluid free-floating particles with an $83 \%$ sensitivity and $63 \%$ specificity, while echogenic fetal thalamus showed $77 \%$ and $79 \%$ for sensitivity and specificity, respectively, for prediction of fetal lung maturity. By using the fetal biometry, we could report that a BPD between 82.8 and $93.5 \mathrm{~mm}, \mathrm{AC}$ between 295 and $322 \mathrm{~mm}$, and FL (62.7-72.1 mm) correlated with mature fetal lungs. Regarding Doppler indices, increased resistive index of umbilical artery $>0.69$ and of pulmonary artery $>0.78$ correlated with neonatal RDS while fetuses with UtA RI values between 0.43 and 0.66 noticed in RDS vs (0.42-0.57) in the non-RDS. MCA RI values are also higher in RDS $0.77-0.88$ vs $0.74-0.79$ in non-RDS.

On the other hand, analysis of colon grading and fetal lung echogenicity in relation to liver showed no significant value in the assessment of fetal lung maturity.

Conclusion: Ultrasound and Doppler showed good predictive value and accuracy and is considered a valuable non-invasive procedure in assessment of fetal lung maturity. However, no single parameter alone could show a definitive sign

Keywords: Fetal lung, Maturity, Doppler, Markers

\footnotetext{
* Correspondence: Reda.alarabawy@yahoo.com

${ }^{2}$ Radiodiagnosis and Medical Imaging Department, Faculty of Medicine,

Tanta University, Tanta, Egypt

Full list of author information is available at the end of the article
}

\section{Springer Open}

(c) The Author(s). 2021 Open Access This article is licensed under a Creative Commons Attribution 4.0 International License, which permits use, sharing, adaptation, distribution and reproduction in any medium or format, as long as you give appropriate credit to the original author(s) and the source, provide a link to the Creative Commons licence, and indicate if changes were made. The images or other third party material in this article are included in the article's Creative Commons licence, unless indicated otherwise in a credit line to the material. If material is not included in the article's Creative Commons licence and your intended use is not permitted by statutory regulation or exceeds the permitted use, you will need to obtain permission directly from the copyright holder. To view a copy of this licence, visit http://creativecommons.org/licenses/by/4.0/. 


\section{Background}

Neonatal respiratory distress syndrome (RDS) remains a major cause of mortality and morbidity in the newly born infants due to immaturity of their lungs. It occurs mainly in newborns who are delivered pre-maturely, and it is associated reversely with gestational age at birth [1].

The decision to continue or delay delivery depends usually on the ability to properly evaluate fetal lung maturity [2].

Lung maturity was commonly assessed roughly by gestational age, and the substitute was amniocentesis but it is invasive, can be performed only by trained persons, is costly, and may have complications [3].

Ultrasound being the most easy, simple, common, non-invasive, and cost-effective tool for routine obstetric scanning [3].

Multiple studies discussed the utility of ultrasound in assessing the fetal lung maturity; ultrasound parameters used were bi-parietal diameter, femur length, epiphyseal centers of the lower limb, placental grading, colon grading, and free-floating particles in the amniotic fluid [35].

Ultrasound can also grade fetal lung morphology and measuring fetal thalamic echogenicity for more attempts to assess fetal lung maturity $[6,7]$.

Pulsed-wave Doppler study, including uterine artery, umbilical artery, fetal middle cerebral artery, and fetal main pulmonary artery are used to settle an association between maternal-fetal Doppler velocimetry parameters and fetal lung maturity indices $[8,9]$.

This work was conducted to assess fetal lung maturity using multiple color Doppler and multiple ultrasound parameters, instead of amniocentesis to reduce the risk of neonatal RDS.

\section{Methods}

The current study was conducted on a total of 200 pregnant women during a period from June 2018 to May 2020 at our university hospitals.

Inclusion criteria are as follows:

Pregnant women with viable fetus aged 30 to 37 weeks of gestation

Exclusion criteria are as follows:

(1) Known chromosomal abnormalities, (2) preexisting maternal medical conditions (e.g., DM, renal diseases, hypertensive disorders), and (3) major congenital anomaly identified after delivery.

This study was designed as a prospective cohort, and it obtained approval from our local Research Ethical Committee. All patients included in this research are above 16 years old and gave written informed consent to publish the data contained within this study.

All included patients were subjected to the following: a. Personal history. Name, age, and parity

b. Sonographic assessment. Ultrasound equipment capable of high-resolution gray-scale, pulsed-wave and color Doppler modes, (GE Healthcare) equipped with a 3-5-MHZ probe transducer

Conventional 2D ultrasound was performed as follows:

1. Bi-parietal diameter $(B P D)$. Bi-parietal diameter was measured from the outer edge of the proximal parietal bone to the inner edge of the distal skull table, in a line perpendicular to the orientation of the cerebral falx.

2. Abdominal circumference $(A C)$. Abdominal circumference was measured at the skin line on a transverse view of the fetal abdomen at the level of the junction of portal sinus, umbilical vein, and fetal stomach when visible.

3. Femur length (FL). Measurement was taken from one end of diaphysis to other.

4. Epiphyseal ossification centers. The femur was identified and its echogenic distal epiphyseal center was located and measured in the axial (anteroposterior) plane. Also, the proximal echogenic tibial epiphysis center is seen at end of tibia.

Measurements were again obtained in the axial (antero-posterior) plane.

5. Placental grading. Grading was done according to Grannum classification [10]:

(a) Grade $0=$ homogenous smooth chorionic plate.

(b) Grade I = undulated chorionic plate with scattered calcifications.

(c) Grade II = incomplete straight line of calcification not reaching the basal plate.

(d) Grade III = indentation and calcification of chorionic plate reaching the basal plate.

\section{Amniotic fluid index and free-floating particles}

The amniotic fluid index AFI was calculated by the fourquadrant technique as the deepest, unobstructed, vertical length of each pocket of fluid was measured in centimeters in each quadrant and then gathered. AFI normally ranged from 8 to 18

Linear densities (Vernix) in the amniotic fluid were noted in four quadrants and measured in millimeters. The amniotic fluid turbidity was recorded depending on the number, size, and distribution of the free-floating particles in four quadrants. They were recognized with their free mobility upon gentle shaking of mothers' abdomen by probe.

\section{Thalamus echogenicity}

Using the ultrasound the bi-parietal diameter (transthalamic plane) was measured in millimeters and the 
state of thalamus echogenicity relative to the brain tissue was reported. The thalamus was considered echogenic if its echogenicity matched the surrounding brain tissue, but it was considered echolucent if it appeared without echoes inside.

\section{Lung to liver echogenicity}

Longitudinal sonograms were performed to include sections of both thorax and abdomen.

Wherever possible, the effect of the ribs was minimized and care was also taken to avoid imaging the lung through the heart or imaging the liver through a fluidfilled fetal stomach since acoustic enhancement posterior to these cystic structures would obviously produce falsely echogenic appearances of the organ parenchyma.

Liver parenchyma should remain relatively constant in its texture throughout the latter half of pregnancy. Echogenicity of the fetal lung was matched against that of the fetal liver and classified as being hypo-echoic, iso-echoic, slightly hyper-echoic, or definitely hyper-echoic in comparison to liver.

\section{Colon grading}

The echogenicity of the meconium inside the colonic cavity was evaluated and graded according to its density. The echogenicity of the colon was assessed and graded in comparison with the echogenicity of the liver.

Grade 0 . The abdominal contents appear uniform and the colon is not identified.

Grade I. The colonic contents are echo-free in appearance echogenicity is essentially identical to that of the bladder and the stomach, and colonic haustra may be identified.

Grade 2. Intermediate. The echogenicity is more than that of the bladder but less than that of the liver.

Grade 3. The echogenicity is essentially equal to the echogenicity of the liver.

\section{Doppler examination}

Flow velocity waveforms were recorded from the umbilical artery umbilical artery $(U A)$ resistive index $(R I)$, uterine artery uterine artery $(U t) R I$, middle cerebral artery middle cerebral artery $(M C A) R I$, and main pulmonary artery main pulmonary artery $(M P A) R I$.

\section{Umbilical artery}

To measure the umbilical artery Doppler, the transducer was placed on the mother's abdomen overlying the fetus and was symmetrically manipulated to obtain the characteristic waveforms at the mid-section of a free loop of the umbilical cord.

\section{Uterine artery}

Color flow imaging was used to identify the bifurcation of the common iliac artery in longitudinal section; then, the probe was moved medially and angled slightly towards the symphysis pubis as uterine artery located just medial to the bifurcation.

The waveforms from both sides of the uterus were recorded and the RI was calculated.

\section{Middle cerebral artery}

A transverse view of the fetal brain was obtained at the level of the bi-parietal diameter (BPD). The transducer was then moved caudally to demonstrate the thalamus clearly. With color flow imaging, the middle cerebral artery (MCA) could be identified as the major anterolateral branch of circle of Willis. The pulsed Doppler sample gate was then placed at the junction of medial third and middle third of this artery. The waveforms from umbilical, uterine, and middle cerebral arteries were obtained. Adjustment of sample volume according to vessel width is advised, color box suitable for view, and also angle is advised less than 60 moreover gain and pulse repetition frequency, even filter are adjusted if needed. All measurements were obtained in the absence of fetal breathing movements.

\section{Main pulmonary artery}

By examining the fetal heart in a systematic manner (the four-chamber view, the outflow tracts, and the threevessel view), at axial view of the thorax, the fetus was at rest without fetal breathing movements, following the MPA until midway between the pulmonary valve and the bifurcation of the right and left branches.

The MPA Doppler waveform appeared with its characteristic needle-like appearance. A small notch of reversed flow is also seen at the end of the systole.

The characteristic shape of MPA waveform differs from the wave of the ductus arteriosus, which is rounded, fuller, and triangular in shape with greater diastolic flow.

After the optimal fetal MPA waveform was obtained, resistance index (RI) was calculated electronically by the machine.

The arterial RI and pulsatility index (PI) were calculated electronically according to the following formulae: $\mathrm{RI}=S-D / S, \mathrm{PI}=S-D / A$

$S$ indicates peak systolic velocity; $D$, end diastolic velocity; and $A$, time averaged velocity

\section{The neonatal outcome}

Upon delivery, the time interval between the ultrasound examination and delivery was recorded and if there was neonatal intensive care unit (NICU) admission for RDS or not. 
Statistical analysis of the collected data was carried out using SPSS software and the following were used:

- Range and mean value \pm standard deviation

- Chi-square

- Monte Carlo test

- Correlation of the parameters with each other

\section{Results}

A total of 200 women were examined in this prospective cohort study, 80 were excluded either due to delivery after 37 weeks of gestation or more than $72 \mathrm{~h}$ after sonography was carried out, administration of antenatal corticosteroids, inability to obtain proper Doppler waveforms (in patients who could not lie still on their backs due to labor pain), or inability of follow up of the neonatal outcome. Thus, a total of 120 women were included and eligible for final analysis.

Maturity of the fetus lung was determined based on the RDS status, where the presence of RDS indicated lung immaturity and vice versa. The majority (83.2\%) of the neonates had favorable outcome with no RDS, whereas 19 of them (15.8\%) had RDS.

\section{Demographic data of the included women regarding} (maternal age, gestational age, gravidity, and parity) (Table 1)

The mean maternal age was 27.89 years \pm 4.37 (range 20-37) years. Mean gestational age was 35.45 weeks \pm 1.72 (range $30-37$ ). Most women had one or more parity, but nulli-parous women represented about one-fourth $(25.8 \%)$ of the study subjects. Twenty-three patients reported a history of abortion.

Table 1 Demographic data of the included women regarding (maternal age, gestational age, gravidity, and parity)

\begin{tabular}{lll}
\hline & Range & Mean \pm SD \\
\hline Maternal age & $20-37$ & $27.98 \pm 4.37$ \\
Gestational age & $30-37$ & $35.45 \pm 1.72$ \\
Gravidity & $1-6$ & $2.17 \pm 1.06$ \\
Parity & $N$ & $\%$ \\
Nullipara & 31 & 25.8 \\
1 & 42 & 35 \\
2 & 27 & 22.5 \\
3 & 11 & 9.2 \\
$\geq 4$ & 9 & 7.5 \\
History of previous abortion & 23 & 19.2 \\
\hline
\end{tabular}

Comparison of the maternal age, gravidity, and parity between the RDS and the non-RDS groups (Table 2)

Results of our study show no statistical significance for comparison between the RDS status regarding the maternal age, gravidity, and parity with a $P$ value $>0.05$. The mean maternal age for the RDS group was 27.84 vs. 28.01 for the non-RDS. The mean gravidity for mothers of RDS fetuses was 2.42 vs. 2.12 for mothers of fetuses with mature lungs. The mean number of parity in mothers of RDS fetuses was 1.42 vs. 1.37 for mothers of fetuses with mature lungs.

\section{Comparison of BPD, AC, and FL between the RDS and the non-RDS (Table 3)}

Fetuses with mature lungs (the non-RDS group), the following diagnostic thresholds predicted lung maturity with $(p$ value $=0.001)$ respectively $\mathrm{BPD} 82.8$ to 93.5 $\mathrm{mm}, \mathrm{AC} 294$ to $322 \mathrm{~mm}$, and FL 62.7 to $73.1 \mathrm{~mm}$. The diagnostic threshold predicts RDS, BPD 77.3-86.3 mm, AC 257-299 $\mathrm{mm}$, and FL 54-65.8 $\mathrm{mm}$.

\section{Comparison of the Doppler waveforms values between the studied RDS and non-RDS fetuses (Table 3 )}

Statistically significant Doppler values between the two groups $(P$ value $=0.001)$.

Regarding the UA RI, a mean value $>0.69$ was found to correlate with delayed lung maturity and neonatal RDS.

The UA PI values were found to be significantly higher in RDS $0.92-1.32$ vs $0.86-1.12$ in non-RDS.

MCA RI values are also higher in RDS $0.77-0.88$ vs $0.74-0.79$ in non-RDS.

MCA PI values showed lower values in RDS 0.89-1.56 vs 1.21-1.61 in non-RDS due to the brain sparing effect.

UtA RI values between the two groups were $0.43-0.66$ in RDS vs $0.42-0.57$ in the non-RDS.

MPA RI showed difference between the two groups of fetuses being $0.74-0.88$ in RDS fetuses vs. $0.71-0.78$ in fetuses with mature lungs.

Table 2 Comparison of the maternal age, gravidity, and parity between the RDS and the non-RDS groups

\begin{tabular}{llllll}
\hline & & Range & Mean \pm S. D & $\boldsymbol{t}$ test & $\boldsymbol{p}$ value \\
\hline Maternal age & RDS & $21-37$ & $27.84 \pm 4.59$ & 0.153 & 0.879 \\
& Non-RDS & $20-37$ & $28.01 \pm 4.36$ & & \\
Gravidity & RDS & $1-6$ & $2.42 \pm 1.43$ & 1.138 & 0.258 \\
& Non-RDS & $1-4$ & $2.12 \pm 0.98$ & & \\
Parity & RDS & $0-4$ & $1.42 \pm 1.22$ & 0.933 & 0.353 \\
& Non-RDS & $0-4$ & $1.37 \pm 1.18$ & & \\
\hline
\end{tabular}


Table 3 Comparison of BPD, AC, FL, and Doppler values between the RDS and the non-RD

\begin{tabular}{llllll}
\hline Parameter & Range & Mean \pm S. D & $\boldsymbol{t}$ test & $\boldsymbol{p}$ value \\
\hline BPD & RDS & $77.3-86.3$ & $81.01 \pm 2.45$ & 8.994 & $0.001^{*}$ \\
& Non-RDS & $82.8-93.5$ & $87.93 \pm 3.18$ & & \\
FL & RDS & $54-65.8$ & $59.84 \pm 3.32$ & 9.839 & $0.001^{*}$ \\
& Non-RDS & $62.7-73.1$ & $68.29 \pm 3.45$ & & \\
AC & RDS & $257-299$ & $280.4 \pm 11.13$ & 12.480 & $0.001^{*}$ \\
& Non-RDS & $294-322$ & $310.2 \pm 9.22$ & & \\
UA RI & RDS & $0.62-0.74$ & $0.69 \pm 0.03$ & 6.731 & $0.001^{*}$ \\
& Non-RDS & $0.58-0.69$ & $0.63 \pm 0.04$ & & \\
UA PI & RDS & $0.92-1.32$ & $1.15 \pm 0.14$ & 8.573 & $0.001^{*}$ \\
& Non-RDS & $0.86-1.12$ & $0.94 \pm 0.09$ & & \\
MCA RI & RDS & $0.77-0.88$ & $0.83 \pm 0.04$ & 10.485 & $0.001^{*}$ \\
& Non-RDS & $0.74-0.79$ & $0.78 \pm 0.01$ & & \\
MCA PI & RDS & $0.89-1.56$ & $1.27 \pm 0.20$ & 6.956 & $0.001^{*}$ \\
& Non-RDS & $1.21-1.61$ & $1.47 \pm 0.10$ & & \\
UtA RI & RDS & $0.43-0.66$ & $0.62 \pm 0.05$ & 6.960 & $0.001^{*}$ \\
& Non-RDS & $0.42-0.57$ & $0.51 \pm 0.04$ & & \\
MPA RI & RDS & $0.74-0.88$ & $0.80 \pm 0.05$ & 8.447 & $0.001^{*}$ \\
& Non-RDS & $0.71-0.78$ & $0.74 \pm 0.02$ & & \\
\hline
\end{tabular}

Correlation between placental grading $(0, \mathrm{I}, \mathrm{II}, \mathrm{III})$ and the neonatal RDS status (Table 4)

Correlation between placental grading by ultrasound and the neonatal RDS status showed a statistical significance with a $P$ value $=0.001$. A total of 31 fetuses had a grade III placenta; only 2 were diagnosed with neonatal RDS, while the remaining 29 had mature lungs.

According to our results, placental grading was sensitive in detection of the fetal lung maturity. It showed a sensitivity of $81 \%$ and specificity of $74 \%$. The PPV was $94 \%$ and NPV was $42 \%$. The accuracy of placental grading by ultrasound for detection of fetal lung maturity was $80 \%$.

\section{Correlation between the presence of free-floating} particles in amniotic fluid and neonatal RDS (Table 4)

The free-floating particles (FFP) sometimes called amniotic fluid particles (AFP) vary in number, size, and distribution (sparse, dense, and very dense/snowstorm).

The AFPs begin to appear at 35 to 36 weeks of gestation. Initially, they are few. The number, size, and distribution of the particles increase gradually and extend to other quadrants [11].

The presence of free-floating particles in amniotic fluid as an ultrasound marker of fetal lung maturity showed a statistical significance with a $P$ value $=0.001$.

FFP was present in a total of 91 fetuses, 7 of them were diagnosed with neonatal RDS, and the remaining
Table 4 Correlation between placental grading and presence of free-floating particles in amniotic fluid and distal femoral epiphyses and proximal tibial epiphyses and thalamic echogenicity and colon grading and lung to liver grading, and neonatal RDS

\begin{tabular}{|c|c|c|c|c|}
\hline Parameter & & RDS & Non-RDS & Total \\
\hline \multicolumn{5}{|c|}{ Placental grading } \\
\hline $0-1$ & $N(\%)$ & $14(73.7)$ & 19 (18.8) & $33(27.5)$ \\
\hline$\|$ & $N(\%)$ & $3(15.8)$ & $53(52.5)$ & $56(46.7)$ \\
\hline III & $N(\%)$ & $2(10.5)$ & $29(28.7)$ & $31(25.8)$ \\
\hline Total & $N(\%)$ & $19(100.0)$ & $101(100.0)$ & $120(100.0)$ \\
\hline Chi-square & \multicolumn{4}{|c|}{$x^{2}=24.169, P$ value $=0.001^{*}$} \\
\hline \multicolumn{5}{|l|}{ FFP } \\
\hline +ve & $N(\%)$ & $7(36.8)$ & $84(83.2)$ & $91(75.8)$ \\
\hline -ve & $N(\%)$ & $12(63.2)$ & $17(16.8)$ & $29(24.2)$ \\
\hline Total & $N(\%)$ & $19(100.0)$ & $101(100.0)$ & $120(100.0)$ \\
\hline Chi-square & \multicolumn{4}{|c|}{$x^{2}=18.727, P$ value $=0.001^{*}$} \\
\hline \multicolumn{5}{|l|}{ DFE } \\
\hline +ve & $N(\%)$ & $3(15.8)$ & $91(90.1)$ & $94(78.3)$ \\
\hline$-v e$ & $N(\%)$ & $16(84.2)$ & $10(9.9)$ & $26(21.7)$ \\
\hline Total & $N(\%)$ & $19(100.0)$ & $101(100.0)$ & $120(100.0)$ \\
\hline Chi-square & \multicolumn{4}{|c|}{$x^{2}=67.553, P$ value $=0.001^{*}$} \\
\hline \multicolumn{5}{|l|}{ PTE } \\
\hline +ve & $N(\%)$ & $1(5.3)$ & $92(91.1)$ & $93(77.5)$ \\
\hline -ve & $N(\%)$ & $18(94.7)$ & $9(8.9)$ & $27(22.5)$ \\
\hline Total & $N(\%)$ & $19(100.0)$ & $101(100.0)$ & $120(100.0)$ \\
\hline Chi-so & \multicolumn{4}{|c|}{$X^{2}=64.487, P$ value $=0.001^{*}$} \\
\hline
\end{tabular}

Thalamic echogenicity

$\begin{array}{lllll}\text { Echogenic } & N(\%) & 5(26.3) & 78(77.2) & 83(69.2) \\ \text { Echolucent } & N(\%) & 14(73.7) & 23(22.8) & 37(30.8) \\ \text { Total } & N(\%) & 19(100.0) & 101(100.0) & 120(100.0)\end{array}$

Chi-square $\quad X^{2}=9.036, P$ value $=0.003^{*}$

Colon

$\begin{array}{lllll}\text { I } & N(\%) & 2(10.5) & 8(7.9) & 10(8.3) \\ \| & N(\%) & 11(57.9) & 36(35.6) & 47(39.2) \\ \text { III } & N(\%) & 6(31.6) & 57(56.4) & 63(52.5) \\ \text { Total } & N(\%) & 19(100.0) & 101(100.0) & 120(100.0)\end{array}$

Chi-square $\quad X^{2}=4.029, P$ value $=0.133$

Lung/liver echogenicity

\begin{tabular}{lllll} 
Hypo-echoic & $N(\%)$ & $7(36.8)$ & $45(44.6)$ & $52(43.3)$ \\
Iso-echoic & $N(\%)$ & $7(36.8)$ & $28(27.7)$ & $35(29.2)$ \\
Hyper-echoic & $N(\%)$ & $5(26.3)$ & $28(27.7)$ & $33(27.5)$ \\
Total & $N(\%)$ & $19(100.0)$ & $101(100.0)$ & $120(100.0)$ \\
Chi-square & $X^{2}=0.687, P$ value $=0.709$ & \\
\hline
\end{tabular}

84 had mature lungs. However, it was absent in $29 \mathrm{fe}-$ tuses, 12 were diagnosed with RDS while the remaining 17 had mature lungs. 
Correlation between the presence of DFE and neonatal RDS status (Table 4)

Distal femoral epiphysis (DFE) showed statistical significance with a $P$ value $=0.001$. DFE was present in a total of 94 cases, 3 of them were diagnosed with RDS, and the remaining 91 had mature lungs, while it was found to be absent in a total of 26 cases, 16 had RDS, and the remaining 10 mature lungs.

\section{Correlation of the presence of the PTE with neonatal RDS} status (Table 4)

Proximal tibial epiphysis (PTE) showed statistically significant with a $P$ value $=0.001$. PTE was present in a total of 93 fetuses, only 1 of them was diagnosed with neonatal RDS, while the remaining 92 had mature lungs. PTE was absent in a total of 27 patients, 18 of them were diagnosed with RDS, and the remaining 9 had mature lungs.

\section{Comparison of sensitivity, specificity, PPV, NPV, and accuracy between FFP, DFE, and PTE}

In this study, the presence of the FFP in amniotic fluid diagnosed by ultrasound was found to be a sensitive indictor of lung maturity, showing sensitivity, specificity, PPV, and NPV of $83 \%, 63 \%, 92 \%$, and $41 \%$, respectively. The accuracy of FFP presence in amniotic fluid for detection of fetal lung maturity was $80 \%$.

The presence of the DFE was more sensitive in the detection of fetal lung maturity. Sensitivity was 90\%, with specificity of $84 \%$. The PPV was $96 \%$ and the NPV was $61 \%$. The accuracy of the DFE presence in the detection of fetal lung maturity was $90 \%$.

While the PTE presence was the most sensitive in detection of fetal lung maturity with $91 \%$ sensitivity, a specificity of $95 \%$. The PPV was $99 \%$ and NPV was $67 \%$ with an accuracy of $92 \%$.

\section{Correlation between thalamic echogenicity and neonatal RDS status (Table 4)}

Thalamic echogenicity showed a statistical significance with a $P$ value $>0.001$. Thalamus was echogenic in a total of 83 fetuses, 5 of which were diagnosed with neonatal RDS, while the remaining 78 had mature lungs. Thalamus was echolucent in a total of 37 fetuses, and 14 of them were diagnosed with neonatal RDS, while the remaining 23 had mature lungs.

\section{Thalamic echogenicity with ultrasound as a predictor of fetal lung maturity}

The current study revealed that thalamic echogenicity was a sensitive predictor of fetal lung maturity, showing a sensitivity of $77 \%$, a specificity of $79 \%$, a PPV of $95 \%$, a NPV of $40 \%$, and an accuracy of $80 \%$.

\section{Correlation between colon grading and neonatal RDS status (Table 4)}

When assessing the correlation of the colon grading to fetal lung maturity and the neonatal RDS status, a total of 10 fetuses had grade I colon, and 2 of them were diagnosed with RDS, while the remaining 8 had mature lungs. A total of 47 fetuses had grade II colon, and 11 of them were diagnosed with neonatal RDS, while the remaining 36 had mature lung. Grade II colon was recorded was recorded in a total of 63 fetuses, and 6 of them were diagnosed with RDS, while the remaining 57 had mature lungs. However, there was no statistical significance with a $P$ value $=0.133$.

\section{Relation between lung/liver echogenicity and neonatal RDS (Table 4)}

Echogenicity of the fetal lungs was matched against that of the fetal liver and was then classified as being hypoechoic, iso-echoic, or hyper-echoic.

The fetal lung/liver echogenicity as an ultrasound marker of fetal lung maturity showed no statistical significance with a $P$ value $>0.05$. The fetal lung/liver echogenicity was hyper-echoic in a total of 33 fetuses, and 5 were diagnosed with neonatal RDS, while the remaining 28 had mature lungs.

\section{Discussion}

The lungs are the last fetal organ essential for extra uterine survival to achieve functional maturity. Moreover, pulmonary surfactant deficiency causes neonatal respiratory distress syndrome [12].

Prenatal diagnosis of lung maturity allow therapeutic protection against possible respiratory distress and permits delivery by planned cesarean section sufficiently early to avoid the possible complications. Some studies of ultrasound prediction of fetal lung maturity compare ultrasound parameters with tests of amniocentesis to assess that sign with lung maturity [7].

The current study was conducted to evaluate accuracy and validity of using ultrasound and Doppler to measure some fetal parameters as indicators of lung maturity in comparison to the neonatal outcome regarding the need for neonatal intensive care unit (NICU) admission due to RDS. These parameters include BPD, FL, AC, placental grading, amniotic fluid vernix (FFP) epiphyseal ossification centers of femur and tibia (DFE and PTE), fetal thalamic echogenicity, colon grading, and lung to liver echogenicity. Doppler parameters also were evaluated in this study including umbilical, middle cerebral artery pulsatility and resistive indices, and uterine and fetal main pulmonary artery resistive indices.

The current study utilized RDS as an indicator of the status of fetal lung maturity, with 19 patients $(15.8 \%$ of neonates had RDS). This percentage decreased with 
advanced gestational age (20\% at 33 weeks, reach $0 \%$ at 37 weeks). Comparable results were reported by Abdulla et al. [13] who found an RDS incidence of $12 \%$ for infants born earlier than 40 weeks gestation versus $0 \%$ for those born after 40 weeks. Similarly, Hibbard et al. [14] reported an incidence of $10.5 \%$ for infants born between 34 and 36 weeks of gestation and only $0.3 \%$ for those born at 38 weeks. Globally, a wide range of RDS incidence has been reported. Compared to the findings of the present study, much less figures were identified by other researchers, where Edwards et al. [15] found an RDS incidence of 7\% and Ghafoor et al. [16] reported even lesser incidence (3.7\%) among neonates at 36 weeks of gestation.

Regarding the Doppler parameters as a marker for fetal lung maturity, we evaluated the values of umbilical artery and our results showed that UA RI $>0.69$ correlated with neonatal RDS.

Scopesi et al. [17] did a study to relate umbilical artery RI Doppler velocimetry to fetal and neonatal distress to establish whether umbilical artery $\mathrm{Rl}$ is a predictor of RDS and assess which fetal parameter is the best predictor of neonatal respiratory distress syndrome. They concluded that increased UA RI values $(>0.70)$ can be considered cut-off value that can predict which fetuses are at low/high risk for respiratory disorders and Doppler velocimetry analysis can provide more reliable information about fetal conditions and predict in which cases timing of delivery and unit care admission should be programmed [17].

In a study by Piazze et al. [9] where fetal lung maturity (FLM) indexes were correlated against Doppler velocimetry values, FLM was assessed by lamellar body count (LBs) in amniotic fluid by amniocentesis. They stated a positive correlation between MCA PI and LBs. MCA PI showed a trend towards lower values in neonates affected by RDS $(1.36 \pm 0.5$ vs. $1.69 \pm 0.4, \mathrm{NS})$.

They also found that a value of mean Ut RI $>0.64$ correlated to a delayed biophysical FLM expressed as a decrease of LBs with a sensitivity of $90.9 \%$ and specificity of $90.3 \%$ [9].

These findings were in agreement with our results as our study also showed that MCA PI had lower values in neonates affected by RDS $(0.89-1.56)$ vs $(1.21-1.61)$ in non-RDS due to the brain sparing effect.

Regarding Ut RI, our results showed higher values in fetuses that were affected by neonatal RDS (0.43-0.66) vs lower values in fetuses without RDS.

In this study, the evaluation of the main pulmonary artery RI showed higher values in fetuses affected by neonatal RDS with a cut point of MPA RI $>0.78$ that correlated with RDS. Laban et al. [12] reported a cut-off value of pulmonary artery (PA) RI $>0.77$ for prediction of neonatal RDS in preterm fetuses and this agrees to our results.

This matched with another study done by Moety et al. [18] mentioned a cut-off value of MPA RI $>0.77$ showed a sensitivity of $67.3 \%$ and specificity of $43.5 \%$ for prediction of neonatal RDS in fetuses delivered in late preterm between 34 and 36 weeks +6 days and early term between 37 and 38 weeks +6 days of gestation.

Conversely, a study by Khanipouyani et al. [1] found no difference in the values of PA RI between RDS fetuses and non-RDS fetuses.

This study assessed the role of epiphyseal ossification centers for prediction of fetal lung maturity. Compared to other parameters the fetal tibia epiphysis was the best indicator of lung maturity followed by the femur epiphysis, where they showed sensitivity, specificity, and accuracy of $91 \%$ vs $90 \%, 95 \%$ vs $84 \%$, and $92 \%$ vs $90 \%$, respectively.

Likewise, Abdulla et al. [13] used the sonographic epiphyseal ossification centers to assess fetal lung maturity, and they correlated these findings with the presence of neonatal RDS. The study reported high sensitivity (95.5\%), specificity (91.7\%), and accuracy (95\%) for fetal tibial epiphysis as a predictor of lung maturity.

These data indicated that visualization and measurement of the fetal knee and shoulder epiphyseal ossification before labor could help to distinguish fetuses with a mature lung amniocentesis profile. Interestingly enough, fetuses that were positive for both distal femur epiphysis and proximal tibia epiphysis had positive outcomes.

However, a number of critical points should be considered. Although the presence of these centers is an indication for maturity of the fetal lung, their absence does not exclude lung maturity. In addition, these epiphyseal centers must be identified with great care to avoid being confused with other adjacent cartilaginous structures. Also, DFE detection of any size may not be associated with fetal lung maturity in complicated pregnancies.

Regarding fetal thalamic echogenicity, the present study reported sensitivity, specificity, and accuracy of $77 \%, 79 \%$, and $80 \%$, respectively, for lung maturity prediction. We observed that thalamic density increased with increased fetus age in our daily practice, so the current study tried to evaluate this sign along with other ultrasound and Doppler parameters as indicators for fetal lung maturity.

Abdullah et al. [13] reported comparable results regarding thalamic echogenicity as an ultrasound marker of fetal lung maturity. Their findings showed sensitivity, specificity, and accuracy of $77.3 \%, 75 \%$, and $77 \%$, respectively.

Moreover, it was consistent with Rasheed et al. [7] who identified fetal lung maturity using thalamic echogenicity with a reported sensitivity, specificity and PPV of $63.33 \%, 86.53 \%$, and $89.6 \%$, respectively. 
The present study used the amniotic fluid vernix for prediction of fetal lung maturity reporting a sensitivity of $83 \%$ and a specificity of $63 \%$.

Comparable results were found by Rasheed et al. [7]. They found that the amniotic fluid vernix as a predictor of lung maturity had a sensitivity of $86.66 \%$ and a specificity of $48.7 \%$.

The role of amniotic fluid particles as a predictor of fetal lung maturity has been studied by several researchers including Ram and Ram [11]. For prediction of RDS, Ram and Ram [11] reported a higher sensitivity (85.74\%) and a lower PPV (66.67\%) compared to our findings. Differences in the procedure used (amniocentesis vs ultrasound) or an inter-observer variation could explain this discrepancy.

Shweni and Moodley [19] reported an established lung maturity in all placental grade II and III fetuses, signifying that placental grading could be used to indicate fetal lung maturity instead of estimating lecithin/sphingomyelin $(\mathrm{L} / \mathrm{S})$ ratio, which will decrease the number of amniocenteses needed.

Loret de Mola et al. [4] reported that placental grade III had a low sensitivity (64\%) and a high specificity (98\%) for mature amniocentesis.

The present study found that grade III placental maturity could predict fetal lung maturity with a sensitivity of $81 \%$, a specificity of $74 \%$, and a PPV of $94 \%$. Comparable results were reported by Abdullah et al. [13]. They showed that for prediction of fetal lung maturity, grade II and III placenta had sensitivity, specificity, and PPV of $60 \%, 75 \%$, and $94 \%$, respectively.

Bowerman et al. [20] established six categories of lung echogenicity relative to liver, ranging from lung much more echogenic than liver to lung less echogenic than liver and might condensed into three with little effect on the results.

Conversely, Cayea et al. [21] found echogenicity of the fetal lung /liver of essentially no value in predicting L/S or phosphatidylglycerol (PG) in amniotic fluid.

Likewise, Fried et al. [22] matched those results, as they found no clinically relevant association between fetal lung echogenicity and gestational age, L/S or presence of PG in amniotic fluid.

Our findings are in close agreement to their findings, as we could establish no significant relation between fetal lung echogenicity to liver and fetal lung maturity or neonatal RDS.

In the current study, fetal biometry as an ultrasound marker for fetal lung maturity revealed threshold values that correlated with lung maturation: BPD 82.8-93.5 $\mathrm{mm}$, FL $62.7-72.1 \mathrm{~mm}$, and AC 294-322 mm.

Chauhan et al. [23] did a study on estimation of fetal parts and correlated the numbers with mature L/S ratio and concluded a diagnostic threshold of BPD 84-92 $\mathrm{mm}$, FL 64-72 $\mathrm{mm}$, and AC $295-350 \mathrm{~mm}$ and those numbers are close to our results.

Regarding the BPD as a marker for fetal lung maturity, correlation of BPD $>90 \mathrm{~mm}$ with a positive shake test (in amniocentesis) showed $100 \%$ predictivity [3].

Loret de Mola et al. [4] reported that a grade 3 colon was $68 \%$ sensitive and $98 \%$ specific for a mature amniocentesis.

Another study by Rezaie Keikhaie et al. [24] classified the fetal intestine into 4 stages. They reported that grade 4 fetal intestine-as a predictor of fetal lung maturityshowed a low sensitivity (62.5\%) and a high specificity (98.9\%).

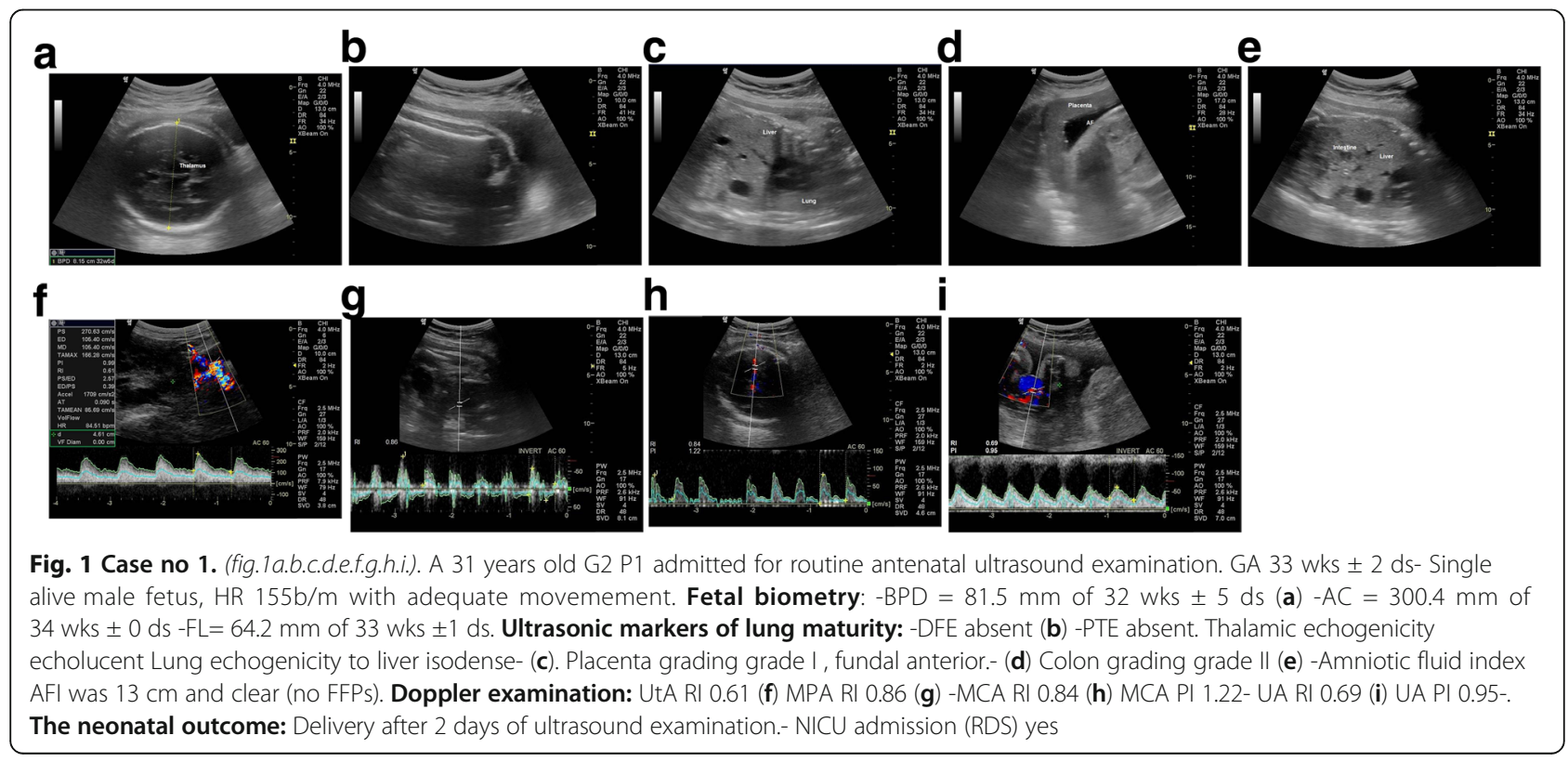




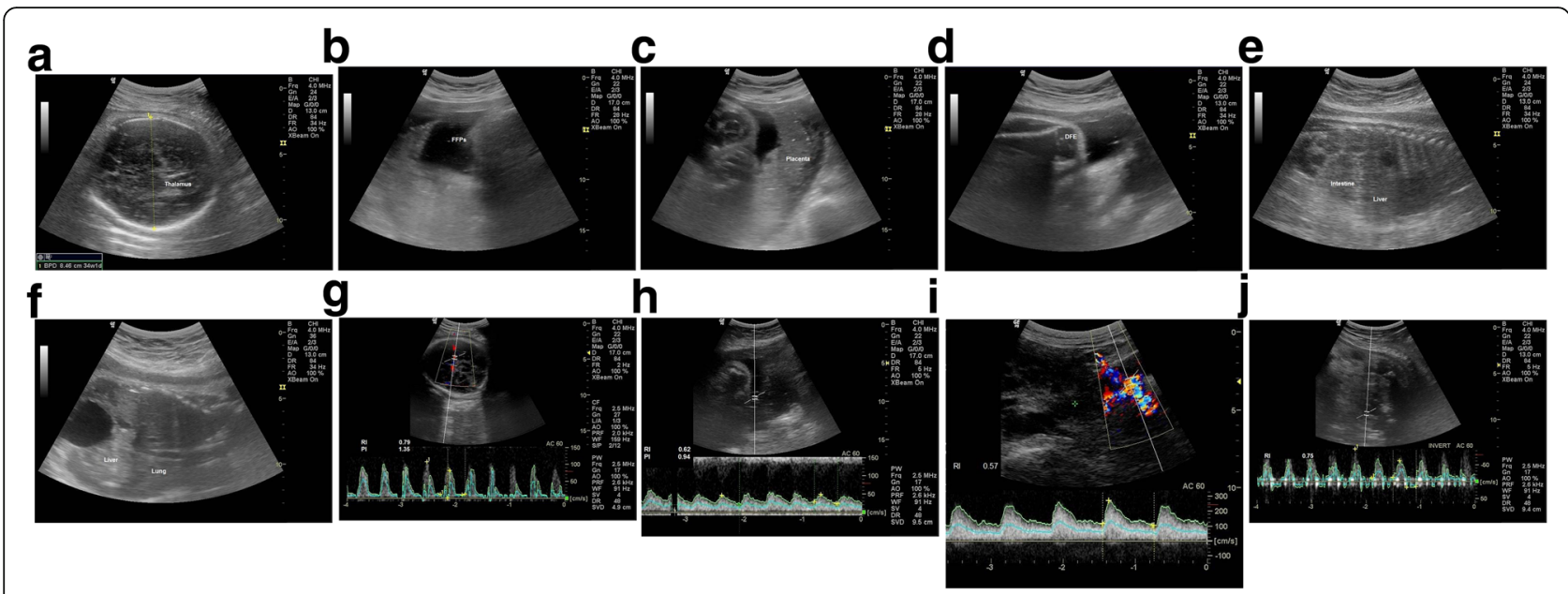

Fig. 2 Case no 2 (fig.2 a.b.c.d.e.f.h.i.j.). A 25 years old PG admitted for routine antenatal ultrasound examination. GA 36 wks \pm 5 ds- Single alive fetus, HR134 b/m with adequate movement. Fetal biometry: $-\mathrm{BPD}=84.6 \mathrm{~mm}$ of $34 \mathrm{wks} \pm 1 \mathrm{ds}(\mathbf{a})$. $-\mathrm{AC}=324 \mathrm{~mm}$ of $36 \mathrm{wks} \pm 3 \mathrm{ds}-\mathrm{FL}=72.1$ $\mathrm{mm}$ of $37 \mathrm{wks}$ ). Ultrasonic markers of lung maturity: Amniotic fluid index AFI was $9 \mathrm{~cm}$ and (FFPs) were clearly seen mobile and swirling motion (b). Placenta grading grade III, fundal posterior (c). -DFE present (d) -PTE absent. Thalamic echogenicity: echogenic. Colon grading grade II (e) Lung echogenicity to liver: hypodense (f). Doppler examination: - MCA RI 0.79 (g) MCA PI 1.35- - UA RI 0.62 (h) UA PI 0.94- - UtA RI 0.57 (i) MPA RI 0.75 (j). The neonatal outcome: Delivery within 24 hours after ultrasound examination.--NICU admission for (RDS) NO

However, in the present study, the assessment of the colon grading as an ultrasound marker of fetal lung maturity showed no significant relation with fetal lung maturity.

The strengths of current study are the following: (1) Nearly the first paper-to the best of our knowledgewhich discusses multiple parameters in a try to assess fetal lung maturity. (2) This study has a prospective design and minimal maternal/fetal co-morbidities in the study subjects, which could increase the applicability and validity of this technique prior to elective delivery of preterm fetuses especially in the event of NICU unavailability. (3) A single sonographer conducted all the ultrasound measurements, thus reducing the inter-observer and intra-observer variation.

(4) The researchers carried out the Doppler and ultrasound measurements within $72 \mathrm{~h}$ of delivery, thereby reducing the variability of the extended time period between measurement and delivery.

The limitations of this study are due to its pilot nature. We recommend the conduction of more studies to validate the results of this study in prediction of RDS in preterm fetuses.

\section{Conclusion}

The current study considers ultrasound and Doppler used for assessment of fetal lung maturity useful noninvasive procedures with good predictive value and accuracy. All of the ultrasound and Doppler parameters tested may be considered predictors of fetal lung maturity with varying degrees of performance.

\section{Abbreviations}

AC: Abdominal circumference; AF GA: Amniotic fluid; AFPs: Amniotic fluid free-floating particles; DFE: Distal femoral epiphyses; PTE: Proximal tibial epiphyses; FFP: Free-floating particles; FLM: Fetal lung maturity; GA: Gestational age; LBs: Lamellar body counts; L/S: Lecithin/sphingomyelin ratio; MCA: Middle cerebral artery; MPA: Main pulmonary artery; NICU: Neonatal intensive care unit; PA: Pulmonary artery;

PG: Phosphatidylglycerol; PI: Pulsatility index; RDS: Respiratory distress syndrome; Rl: Resistive index; UA: Umbilical artery; Ut/UtA: Uterine artery

\section{Acknowledgements}

Not applicable

\section{Authors' contributions}

RAK: Care of the patients and aid in writing and statistics. AZA: Aid in revision of writing and cases. MHE: Final revision and supervision. RAA: Had the idea of research and revision and aid in writing and publishing. All authors read and approve the final manuscript.

\section{Funding}

Not applicable

\section{Availability of data and materials}

The datasets generated or analyzed during current study are not publicly available as the license taken for the current study is only not public, but the data are available on reasonable request from corresponding author.

\section{Ethics approval and consent to participate}

This study was conducted according to the guidelines of the ethics committee of our university and was approved by our institutional review board.

All patients included in this research are above 16 years old and gave written informed consent to publish the data contained within this study.

The committee reference number 32354/05/18

Consent for publication

Not applicable 


\section{Competing interests}

The authors declare no competing interests.

\section{Author details}

'Radiodiagnosis and Medical Imaging Department, Elmenshawy General Hospital, Tanta, Egypt. ${ }^{2}$ Radiodiagnosis and Medical Imaging Department, Faculty of Medicine, Tanta University, Tanta, Egypt.

Received: 29 September 2020 Accepted: 3 February 2021

Published online: 16 March 2021

\section{References}

1. Khanipouyani F, Abbasalizade F, Abbasalizade S, Fardiazar Z, Ghaffari S, Sarbakhshi $P$, editors. Predicting fetal lung maturity using the fetal main pulmonary artery doppler indices. 2016.

2. Ghorayeb SR, Bracero LA, Blitz MJ, Rahman Z, Lesser ML (2017) Quantitative ultrasound texture analysis for differentiating preterm from term fetal lungs. J Ultrasound Med 36(7):1437-1443

3. Pallavi L, Sushil K, Lakhkar DL, Soniya D, Abhijeet I, Pooja S (2017) Assessment of fetal lung maturity by ultrasonography. Ann Int Med Den Res 3(4):RD01-RD04

4. Loret de Mola JR, Judge N, Entsminger C, DeViney M, Muise KL, Duchon MA (1998) Indirect prediction of fetal lung maturity. Value of ultrasonographic colonic and placental grading. J Reprod Med 43(10):898-902

5. Tahilramaney MP, Platt LD, Golde SH (1991) Use of femur length measured by ultrasonography to predict fetal maturity. J Perinatol 11(2):157-160

6. Palacio M, Bonet-Carne E, Cobo T, Perez-Moreno A, Sabrià J, Richter J et al (2017) Prediction of neonatal respiratory morbidity by quantitative ultrasound lung texture analysis: a multicenter study. Am J Obstet Gynecol 217(2):196.e191-196.e114

7. Rasheed F, Al-Sattam Z, Hussain S (2012) Evaluation of thalamus echogenicity by ultrasound as a marker of fetal lung maturity. Open J Obstet Gynecol 2:270-275

8. Azpurua H, Norwitz ER, Campbell KH, Funai EF, Pettker CM, Kleine M et al (2010) Acceleration/ejection time ratio in the fetal pulmonary artery predicts fetal lung maturity. Am J Obstet Gynecol 203(1):40.e41-40.e48

9. Piazze JJ, Anceschi MM, Picone G, Cerekja A, La Torre R, Cosmi EV (2003) Association between maternal-fetal Doppler velocimetry and fetal lung maturity. J Perinat Med 31(6):484-488

10. Grannum PA, Berkowitz RL, Hobbins JC (1979) The ultrasonic changes in the maturing placenta and their relation to fetal pulmonic maturity. Am J Obstet Gynecol 133(8):915-922

11. Ram SHS, Ram D (2010) Role of echogenic amniotic fluid particles and optical density in prediction of respiratory distress syndrome and labor. Internet J Med Update 5(1):3-11

12. Laban M, Mansour GM, El-Kotb A, Hassanin A, Laban Z, Saleh A (2019) Combined measurement of fetal lung volume and pulmonary artery resistance index is more accurate for prediction of neonatal respiratory distress syndrome in preterm fetuses: a pilot study. J Matern Fetal Neonatal Med 32(4):626-632

13. Abdullah T, Hassan QA, Ameen BA (2018) Prediction of fetal lung maturity by ultrasonic thalamic echogenicity and ossification centers of fetal femur and tibia. Ital J Gynaecol Obstet 30:29-36

14. Hibbard JU, Wilkins I, Sun L, Gregory K, Haberman S, Hoffman M et al (2010) Respiratory morbidity in late preterm births. JAMA 304(4):419-425

15. Edwards MO, Kotecha SJ, Kotecha S (2013) Respiratory distress of the term newborn infant. Paediatr Respir Rev 14(1):29-36 quiz 36-27

16. Ghafoor T, Mahmud S, Ali S, Dogar SA (2003) Incidence of respiratory distress syndrome. J Coll Physicians Surg Pak 13(5):271-273

17. Scopesi F, Gazzolo D, Cortez R, Nigro M, Pittaluga C, Russo A et al (1994) Neonatal respiratory distress syndrome screening by behavioural state and umbilical artery Doppler velocimetry. J Perinat Med 22(Suppl 1):176-178

18. Moety GA, Gaafar HM, El Rifai NM (2015) Can fetal pulmonary artery Doppler indices predict neonatal respiratory distress syndrome? J Perinatol 35(12):1015-1019

19. Shweni PM, Moodley SC (1986) Placental grading by ultrasonography as an index of fetal maturity. Its application to the problem of elective caesarean section. S Afr Med J 70(9):525-528

20. Bowerman RA, Dodge KM, Carson PL (eds) (1984) Ultrasonic prediction of pulmonary maturity: correlation with $\mathrm{L} / \mathrm{S}$ ratio. The annual meeting of the American Institute of Ultrasound in Medicine. Kansas City, Wiley
21. Cayea PD, Grant DC, Doubilet PM (eds) (1984) Inability of current clinical ultrasound equipment to predict fetal lung maturity. The annual meeting of the American Institute of Ultrasound in Medicine. Kansas City, Wiley

22. Fried AM, Loh FK, Umer MA, Dillon KP, Kryscio R (1985) Echogenicity of fetal lung: relation to fetal age and maturity. AJR Am J Roentgenol 145(3):591-594

23. Chauhan SP, Magann EF, Morrison JC, Gunter AD, Whitworth NS, Devoe LD (2000) Sonographic measurements of fetal parts to predict pulmonary maturity among twins and singletons. J Miss State Med Assoc 41(3):516-520

24. Rezaie Keikhaie K, Rezaie Keikhaie K, Rezaie Keikhaie K, Rezaie Keikhaie K, Kahkhaie KR, Mohammadi N et al (2017) Relationship between ultrasonic marker of fetal lung maturity and lamellar body count. J Natl Med Assoc 109(4):294-298

\section{Publisher's Note}

Springer Nature remains neutral with regard to jurisdictional claims in published maps and institutional affiliations.

\section{Submit your manuscript to a SpringerOpen ${ }^{\circ}$ journal and benefit from:}

- Convenient online submission

- Rigorous peer review

- Open access: articles freely available online

High visibility within the field

- Retaining the copyright to your article

Submit your next manuscript at $\boldsymbol{\nabla}$ springeropen.com 